\title{
Mortalidade por hepatite viral B no Brasil, 2000-2009
}

\author{
Hepatitis B mortality in Brazil, \\ 2000-2009
}

\author{
1 Programa de Treinamento \\ em Epidemiologia Aplicada \\ aos Serviços do SUS, \\ Ministério da Saúde, Brasília, \\ Brasil. \\ 2 Departamento de DST/AIDS \\ e Hepatites Virais, Secretaria \\ de Vigilância em Saúde, \\ Ministério da Saúde, Brasília, \\ Brasil. \\ Correspondência \\ M. C. Tauil \\ Programa de Treinamento em \\ Epidemiologia Aplicada aos \\ Serviços do SUS, Secretaria \\ de Vigilância em Saúde, \\ Ministério da Saúde. \\ SHIN QI 11, conjunto 4, casa \\ 20, Brasília, DF 71515-740, \\ Brasil. \\ mctauil@hotmail.com
}

\begin{abstract}
This descriptive study focused on the mortality profile associated with hepatitis B virus ( $H B V$ ) in Brazil as a whole and by region, based on data from the Mortality Information System (MIS). The study sample consisted of deaths from $\mathrm{HBV}$ recorded in the MIS from January 1, 2000, to December 31, 2009. The crude mortality rate remained constant in the country; the proportion of hepatocellular carcinoma with HBV as the associated cause was no greater than $7 \%$. The standardized mortality rate was highest in the North of Brazil, and the proportional mortality rate was higher in males. In 2009, the potential years of life lost (PYLL) were highest in males in the 50-59year age bracket and in females in the 40-49-year bracket. The largest increase in PYLL occurred in males 60 to 69 years of age. The study emphasizes the importance of scaling up preventive measures against $\mathrm{HBV}$, in addition to expanding access to early diagnosis in order to reduce HBV mortality in the coming decades.
\end{abstract}

Hepatitis B; Information Systems; Mortality

\author{
Márcia de Cantuária Tauil 1 \\ Thiago Rodrigues de Amorim 2 \\ Gerson Fernando Mendes Pereira 2 \\ Wildo Navegantes de Araújo 1
}

\section{Introdução}

A hepatite viral B constitui um relevante problema de saúde pública, correspondendo à causa mais frequente de hepatite crônica, cirrose e carcinoma hepatocelular (CHC) ${ }^{1}$. Apresenta amplo espectro clínico, desde infecções assintomáticas, oligossintomáticas, até formas fulminantes 2 . A infectividade do vírus da hepatite B (VHB) é de 50 a 100 vezes maior do que a do HIV 1, e a susceptibilidade à infecção é universal. Suas vias de transmissão consistem em sexual, parenteral e vertical ${ }^{3}$.

De maneira geral, sua evolução é lenta, insidiosa e progressiva, o que dificulta a realização de estudos que permitam conhecer a real magnitude dessa doença. Além disso, o fato de a morte por câncer de fígado em portadores do VHB ser codificada como "câncer de fígado" e não como "hepatite B" 4 agrava ainda mais a subnotificação do óbito por essa doença 5 .

A imunidade para a hepatite B pode ocorrer por meio de infecção passada ou vacinação 6,7 . No Brasil, os estudos realizados a partir da década de 90 indicam redução na endemicidade da infecção pelo VHB 8,9,10,11. Isso se deve, provavelmente, à instituição, em 1998, da vacinação universal contra hepatite B para menores de um ano e a posterior ampliação, a partir de 2001, de sua oferta gratuita para menores de 20 anos 12 . Além disso, a Portaria $n^{\circ}$. 1.376/93, reforçada 
pela Resolução $n^{o} .343 \mathrm{MS} / 2001$, determinam a realização de testes de triagem sorológica nos serviços de hemoterapia para hepatite $B$, entre outras doenças 13.

De acordo com a Organização Mundial da Saúde (OMS), estima-se que dois bilhões de pessoas no mundo já foram infectados pelo VHB; destas, 400 milhões permaneceram cronicamente infectados. Em países da Europa, a prevalência de HBsAg varia de menos de $2 \%$ a $7 \%$. No Brasil, a OMS classifica a Região Norte como de alta endemicidade (prevalência de HBsAg maior que $8 \%$ ), e as demais regiões, como de intermediária endemicidade (prevalência de HBsAg entre $2 \%$ e $8 \%$ ). Todavia, os resultados do estudo de base populacional das infecções pelos vírus das hepatites A, B e C sugeriram a ocorrência de baixa endemicidade (menor que $2 \%$ ) da infecção pelo VHB no conjunto das capitais de cada macrorregião e no Distrito Federal. Para o marcador HBsAg, a prevalência global referente ao conjunto das capitais do Brasil foi de $0,4 \%$, sendo de $0,1 \%$ na faixa etária de 10 a 19 anos e de $0,6 \%$ para os grupos de 20 a 69 anos 14 .

Atualmente não existem informações consolidadas referentes à tendência da mortalidade por hepatite B no Brasil. O objetivo deste trabalho foi descrever o perfil dessa mortalidade com base nos dados disponíveis em um sistema oficial de informação existente no país.

\section{Métodos}

Trata-se de estudo descritivo da distribuição espacial, temporal, por sexo e grupo etário dos óbitos por hepatite B registrados no Sistema de Informações sobre Mortalidade (SIM), durante o período de 1o de janeiro de 2000 a 31 de dezembro de 2009. A área investigada foi o território brasileiro, categorizado em cinco regiões (Norte, Nordeste, Sudeste, Sul e Centro-oeste). O Brasil possui uma área de $8.514 .215,3 \mathrm{~km}^{2}$ e tem uma população estimada para o ano de 2009 de 191.481.045 habitantes (Instituto Brasileiro de Geografia e Estatística. http://www.ibge.gov.br/ home/default.php, acessado em 27/Set/2010).

Foram considerados óbitos por hepatite B aqueles que apresentavam pelo menos uma das seguintes codificações para causa básica, segundo a 10a revisão da Classificação Internacional de Doenças (CID-10) 15: B16 (Hepatite aguda B), B16.0 (Hepatite aguda B com agente Delta, coinfecção, com coma hepático); B16.1 (Hepatite aguda B com agente Delta, coinfecção, sem coma hepático), B16.2 (Hepatite aguda B sem agente Delta, com coma hepático), B16.9 (Hepatite aguda B sem agente Delta e sem coma hepático),
B17.0 (superinfecção Delta aguda de portador de hepatite B); B18.0 (Hepatite viral crônica B com agente Delta); B18.1 (Hepatite crônica viral B sem agente Delta). Incluíram-se também os óbitos que tiveram como causa básica os códigos C22.0 (Hepatocarcinoma) ou K74.6 (Cirrose hepática) e alguns dos códigos da CID 10 acima como causa associada. Além disso, o total de óbitos por hepatocarcinoma foi utilizado para se verificar em que proporção estes tiveram hepatite B como causa associada.

Para a análise de dados, foi calculada a taxa bruta de mortalidade por hepatite B por 100 mil habitantes no Brasil, por ano. As populações utilizadas como denominadores para os cálculos de indicadores foram as do censo de 2000, das estimativas e das projeções populacionais anuais do período do estudo, disponibilizadas pelo Instituto Brasileiro de Geografia e Estatística (http:/ / www.ibge.gov.br/home/default.php, acessado em 27/Set/2010). Também se calculou a proporção de óbitos por hepatocarcinoma com hepatite B como causa associada, a taxa de mortalidade padronizada pela população do Brasil em 2000, por região, e a mortalidade proporcional por hepatite B, por região e grupo etário. A mortalidade proporcional foi calculada até o ano de 2008, pois o número total de óbitos somente estava disponível até esse ano.

Os cálculos de anos potenciais de vida perdidos (APVP) foram realizados por meio de uma adaptação da proposta de Romeder \& McWhinnie 16,17. Inicialmente, tomou-se a diferença entre o ponto médio do grupo etário em relação ao limite de idade para os homens e para as mulheres e multiplicou-se pelo número de óbitos ocorridos em cada grupo etário; em seguida, os subtotais foram somados. Para o cálculo das taxas de APVP (TAPVP), dividiram-se os anos potenciais de vida perdidos em determinado grupo etário pela população desse grupo, multiplicado por 100 mil habitantes (Ministério da Saúde. http://www2.datasus.gov.br/DATASUS/index. php, acessado em 27/Set/2010). Foram excluídos os óbitos em menores de um ano e o limite de idade utilizado para homens e mulheres foi 70 anos.

Os dados foram analisados com auxílio dos softwares PASW 18.0 (IBM, Nova York, Estados Unidos) e Excel 2007 (Microsoft Corp., Estados Unidos).

Para o uso das bases de dados, foram solicitadas as autorizações de acesso às instituições gestoras e responsáveis pela guarda, sendo mantida a confidencialidade das informações. Esse trabalho foi aprovado pela Comissão Nacional de Ética em Pesquisa (parecer no ${ }^{\circ}$ 16255). 


\section{Resultados}

Foram registradas 5.441 mortes por hepatite B no período de 2000 a 2009, com uma mediana de 527,5 óbitos, variando de 453 a 684 . De acordo com a Tabela 1, a taxa bruta de mortalidade pela doença no Brasil variou de 0,3 a 0,4 por $100 \mathrm{mil}$ habitantes, e a maior proporção de óbitos $(6,4 \%)$ por hepatocarcinoma com hepatite B como causa associada ocorreu em 2001.

No ano de 2008, a taxa de mortalidade padronizada por hepatite B foi maior na Região Norte, 0,9 por 100 mil habitantes, seguida da Região Centro-oeste, 0,5 por 100 mil habitantes. A Região Nordeste foi a que apresentou as menores taxas, sendo de 0,1 por 100 mil habitantes de 2000 a 2005 e de 0,2 por 100 mil habitantes de 2006 a 2009 (Figura 1).

A mortalidade proporcional foi maior no sexo masculino, variando de $69 \%$ a $79 \%$. No sexo feminino variou de $26 \%$ a $31 \%$ (Figura 2 ).

Com relação ao grupo etário, na Região Norte a mortalidade proporcional foi maior no grupo de 30 a 39 anos, enquanto nas regiões Nordeste e Centro-oeste, no grupo de 40 a 49 anos. Já nas regiões Sudeste e Sul, a mortalidade proporcional foi maior no grupo de 50 a 59 anos (Tabela 2).

Os APVP no sexo masculino foram maiores no grupo etário de 40 a 49 anos em 2000 e no grupo de 50 a 59 anos em 2009. No sexo feminino, os APVP, no ano 2000, foram maiores no grupo de 30 a 39 anos e, em 2009, no grupo de 40 a 49 anos. Conforme a Tabela 3, houve aumento da TAPVP (total) no sexo masculino e não houve variação no sexo feminino. Ocorreu aumento nas TAPVP nos grupos etários de 50 a 59 anos e de 60 a 69 anos, no sexo masculino, e nos grupos etários de 40 a 49 e de 50 a 59 anos, no sexo feminino. Para os grupos etários de 20 a 29 e de 30 a 39 anos, houve redução da TAPVP para ambos os sexos.

\section{Discussão e conclusões}

A taxa bruta de mortalidade por hepatite B no Brasil manteve-se constante no período, sendo menor do que a de outras doenças, como, por exemplo, a tuberculose (2,8 por 100 mil habitantes em 2004) 18. Possíveis explicações para essa constância podem estar relacionadas ao início da vacinação para menores de 20 anos, que ocorreu somente a partir de 2001, e também às coberturas vacinais abaixo do recomendado (Ministério da Saúde. http://tabnet.datasus.gov.br/cgi/ tabcgi.exe?pni/cnv/cpniuf.def, acessado em 15/ Dez/2010).

A alta taxa de mortalidade padronizada detectada na Região Norte pode se dever à elevada prevalência de hepatite $\mathrm{B}$ nessa região, além da coinfecção/superinfecção por hepatite D, principalmente entre as populações indígenas, conforme descrito em vários estudos 10,19,20,21. Essa região também apresentou maior concentração de óbitos em um grupo etário mais novo. Uma possível explicação pode estar relacionada

Tabela 1

Óbitos por hepatite B e por hepatocarcinoma com hepatite B como causa associada. Brasil, 2000-2009.

\begin{tabular}{cccccc}
\hline Ano & $\begin{array}{c}\text { Óbitos por hepatite } \\
\text { B }\end{array}$ & TM * & $\begin{array}{c}\text { Óbitos por } \\
\text { hepatocarcinoma ** }\end{array}$ & $\begin{array}{c}\text { Total de óbitos por } \\
\text { hepatocarcinoma }\end{array}$ \\
\hline 2000 & 453 & 0,3 & 25 & 504 & 5,0 \\
2001 & 510 & 0,3 & 37 & 582 & 6,4 \\
2002 & 468 & 0,3 & 30 & 996 & 3,0 \\
2003 & 481 & 0,3 & 35 & 1.053 & 3,3 \\
2004 & 488 & 0,3 & 45 & 1.185 & 3,8 \\
2005 & 545 & 0,3 & 49 & 1.39 & 4,1 \\
2006 & 617 & 0,3 & 65 & 1.275 & 4,9 \\
2007 & 629 & 0,3 & 57 & 1.376 & 4,5 \\
2008 & 684 & 0,4 & 65 & 1.438 & 4,7 \\
2009 & 566 & 0,3 & 60 & 10.922 & 4,2 \\
Total & 5.441 & 0,3 & 468 & & 4 \\
\hline
\end{tabular}

* Taxa bruta de mortalidade por hepatite B por 100 mil habitantes.

** Óbitos por hepatocarcinoma com hepatite B como causa associada.

*** Proporção de óbitos por hepatocarcinoma com hepatite B como causa associada. 
Taxa de mortalidade por hepatite B padronizada pela população do ano 2000, por 100 mil habitantes. Brasil, 2000-2009.

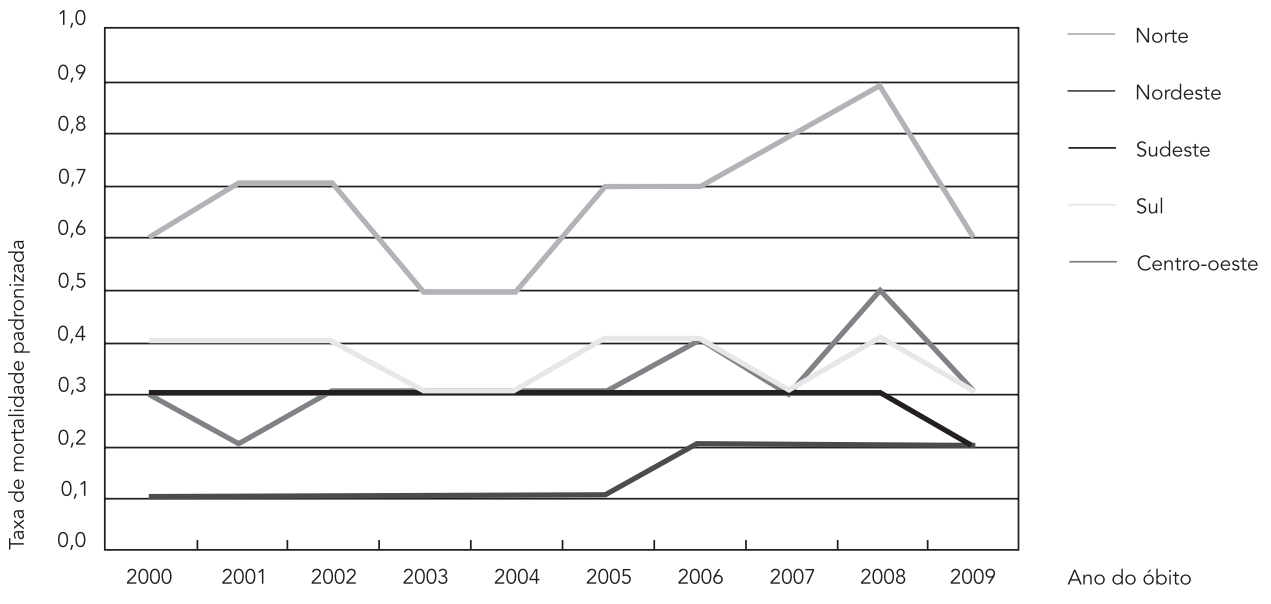

Figura 2

Mortalidade proporcional por hepatite B segundo sexo. Brasil, 2000-2009.

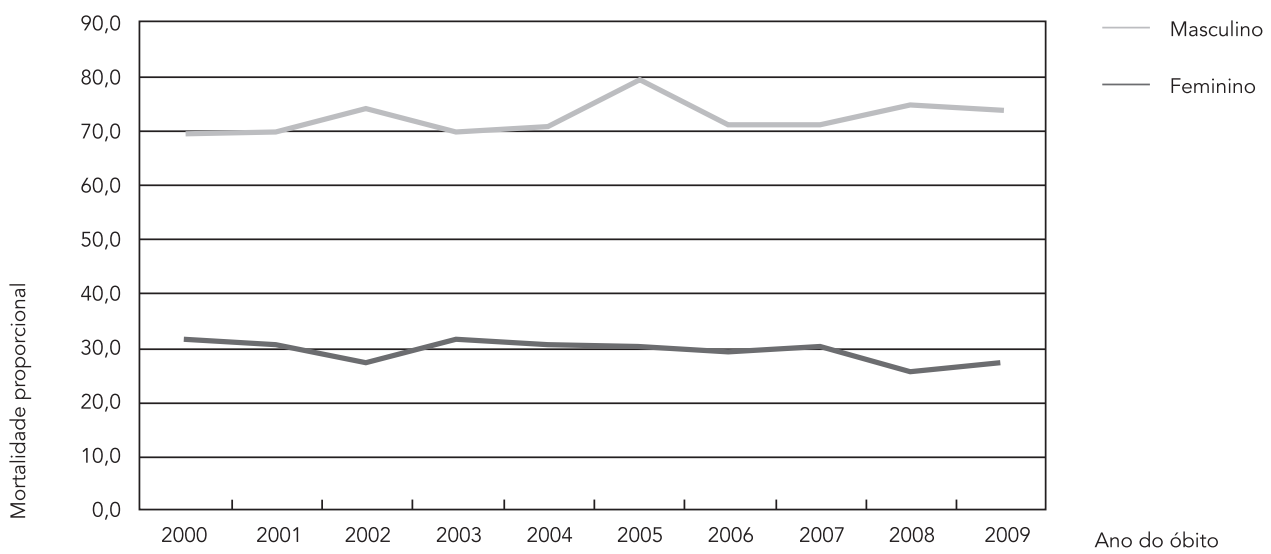

à superinfecção pela hepatite $\mathrm{D}$, que tem sido apontada como causa de diversos surtos de hepatite grave na bacia amazônica brasileira 20 .

No presente estudo, a proporção de óbitos por hepatocarcinoma que tem a hepatite B como causa associada foi maior do que em uma pesquisa realizada em 1990, nos Estados Unidos, a qual encontrou 3\% (136/4.543). Porém, os autores relatam que muitos pacientes com hepatocarcinoma são infectados com hepatite B, mas nem sempre são testados para esta doença 22. Por outro lado, em uma investigação com 236 indivíduos com hepatocarcinoma, tendo como critério de inclusão idade acima de 16 anos, em 19 centros médicos de oito estados do Brasil, identificou-se que 39\% dos casos tinham hepatite B ${ }^{23}$. Além disso, de acordo com artigo sobre prevenção de hepatocarcinoma, 55\% dos casos 
Mortalidade proporcional por hepatite B (por 100 mil óbitos) segundo grupo etário. Regiões do Brasil, 2000-2008.

\begin{tabular}{lccccc}
\hline Grupo etário (anos) & Norte & Nordeste & Sudeste & Sul & Centro-oeste \\
\hline$<5$ & 10 & 9 & 3 & 2 & 8 \\
$5-9$ & 163 & 34 & 13 & 35 & 29 \\
$10-19$ & 206 & 23 & 16 & 44 & 35 \\
$20-29$ & 251 & 25 & 33 & 39 & 60 \\
$30-39$ & 411 & 43 & 85 & 96 & 92 \\
$40-49$ & 350 & 64 & 102 & 137 & 149 \\
$50-59$ & 230 & 53 & 103 & 148 & 115 \\
$\geq 60$ & 64 & 17 & 37 & 48 & 38 \\
\hline
\end{tabular}

Tabela 3

Anos potenciais de vida perdidos por hepatite B, por sexo e grupo etário. Brasil, 2000 e 2009.

\begin{tabular}{|c|c|c|c|c|c|c|c|}
\hline \multirow[t]{2}{*}{ Grupo etário (anos) } & \multicolumn{3}{|c|}{2000} & \multicolumn{3}{|c|}{2009} & \multirow{2}{*}{$\begin{array}{l}\text { Variação \% } \\
\text { das TAPVP }\end{array}$} \\
\hline & APVP & $\%$ & TAPVP * & APVP & $\%$ & TAPVP * & \\
\hline \multicolumn{8}{|l|}{ Sexo masculino } \\
\hline $1-4$ & 203 & 3 & 3 & 68 & 1 & 1 & $-66,7$ \\
\hline $5-9$ & 252 & 4 & 3 & 0 & 0 & 0 & $-100,0$ \\
\hline $10-19$ & 111 & 2 & 1 & 222 & 3 & 1 & 0,0 \\
\hline $20-29$ & 910 & 15 & 6 & 865 & 12 & 5 & $-16,7$ \\
\hline $30-39$ & 1.172 & 20 & 10 & 1.207 & 17 & 9 & $-10,0$ \\
\hline $40-49$ & 1.683 & 28 & 18 & 2.015 & 29 & 17 & $-5,6$ \\
\hline $50-59$ & 1.318 & 22 & 22 & 2.046 & 29 & 24 & 9,1 \\
\hline $60-69$ & 264 & 4 & 7 & 572 & 8 & 12 & 71,4 \\
\hline Total & 5.912 & 100 & 7 & 6.994 & 100 & 8 & 14,3 \\
\hline \multicolumn{8}{|l|}{ Sexo feminino } \\
\hline $1-4$ & 0 & 0 & 0 & 0 & 0 & 0 & 0,0 \\
\hline $5-9$ & 63 & 2 & 1 & 0 & 0 & 0 & $-100,0$ \\
\hline $10-19$ & 500 & 19 & 3 & 222 & 9 & 1 & $-66,7$ \\
\hline $20-29$ & 410 & 16 & 3 & 364 & 15 & 2 & $-33,3$ \\
\hline $30-39$ & 781 & 30 & 6 & 533 & 23 & 4 & $-33,3$ \\
\hline $40-49$ & 408 & 16 & 4 & 612 & 26 & 5 & 25,0 \\
\hline $50-59$ & 279 & 11 & 4 & 496 & 21 & 5 & 25,0 \\
\hline $60-69$ & 182 & 7 & 4 & 132 & 6 & 2 & $-50,0$ \\
\hline Total & 2.622 & 100 & 3 & 2.359 & 100 & 3 & 0,0 \\
\hline
\end{tabular}

APVP: anos potenciais de vida perdidos; TAPVP: taxa de anos potenciais de vida perdidos.

* Para o cálculo da TAPVP, utilizou-se a população por grupo etário disponível na página da Internet do DATASUS.

Taxa por 100.000 habitantes.

são secundários à infecção pelo VHB 24. Portanto, é bem provável que haja subnotificação de óbitos por esta doença no país.

Segundo artigos relacionados à qualidade das estatísticas vitais, o SIM vem melhorando acentuadamente, seja quanto à cobertura, seja quan- to à qualidade de seus dados 25,26. Apesar disso, a subnotificação de óbitos por hepatite B pode ocorrer, principalmente, nas regiões brasileiras menos favorecidas, pela dificuldade de acesso ao diagnóstico e pela não codificação dessa doença na declaração de óbito. 
O predomínio do sexo masculino na mortalidade proporcional e o aumento da TAPVP neste sexo podem sugerir maior exposição deste grupo ao vírus, possivelmente em virtude do maior uso de drogas injetáveis 27 , porém outros estudos são necessários para investigar tais hipóteses nesta população. Provavelmente, a infecção pelo VHB vem ocorrendo em faixas etárias mais precoces, levando-se em conta que o óbito por hepatite B é um evento tardio 28 . Isso aponta para a importância das medidas de prevenção, principalmente a vacinação, redução do uso de drogas injetáveis, uso de preservativo no ato sexual pelos jovens e cuidados na transfusão de sangue.

De acordo com estudo realizado em Pernambuco em 2005, os APVP por AIDS nesse estado foram de 12.74429 , maiores do que os encontrados por hepatite B, 9.353, para o Brasil em 2009. Outro estudo sobre mortalidade por hepatite B na Espanha, no ano 2000, refere 880 APVP por hepatite B para homens e 295 APVP por hepatite B para mulheres, sendo esses valores bem menores do que os encontrados no nosso estudo 28 . Talvez, os APVP na Espanha reflitam os resultados do programa de imunização desse país, aceito como modelo para outras nações 6 .

\section{Resumo}

Este trabalho trata-se de estudo descritivo, cujo objetivo foi descrever o perfil de mortalidade pelo vírus da hepatite B (VHB) no Brasil e regiões, com base nos dados disponíveis no Sistema de Informações sobre Mortalidade (SIM). Constituíram a população de estudo os óbitos pelo VHB registrados no SIM durante o período de 1o de janeiro de 2000 a 31 de dezembro de 2009. A taxa bruta de mortalidade no país permaneceu constante; a proporção de óbitos por hepatocarcinoma com VHB como causa associada não ultrapassou $7 \%$. A taxa de mortalidade padronizada foi maior na Região Norte, e a mortalidade proporcional foi maior no sexo masculino. Em 2009, os anos potenciais de vida perdidos (APVP) no sexo masculino foram maiores no grupo etário de 50 a 59 anos; no sexo feminino, no grupo de 40 a 49 anos. O maior aumento da taxa de APVP ocorreu no sexo masculino (60 a 69 anos). Esta pesquisa reforça a importância de se aumentarem as medidas de prevenção contra a hepatite $B$, além de se ampliar o acesso ao diagnóstico precoce para que haja a redução da mortalidade nas próximas décadas.
O presente trabalho apresenta as limitações relacionadas ao uso de dados secundários, os quais, em geral, são subestimados. No caso do SIM, também se deve considerar que há uma proporção de mortes por causas mal definidas. Por outro lado, trata-se de uma fonte de registro padronizada, além de ser a primeira abordagem sobre o tema para todo o Brasil. Com relação aos APVP, houve subestimação, já que os óbitos abaixo de um ano e acima do limite de idade estabelecido não entraram no cálculo. Nesses extremos de idade foram excluídos 116 (16\%) óbitos do sexo masculino e 77 (26\%) do feminino. Por outro lado, os APVP podem ter sido superestimados, pois os indivíduos não necessariamente iriam sobreviver até o limite de vida esperado, havendo a possibilidade de morrerem por outras causas evidentemente não computadas.

De acordo com este estudo, a mortalidade por hepatite B no Brasil, no período de 2000 a 2009, manteve-se constante. A Região Norte destacou-se com as maiores taxas, o sexo masculino apresentou a maior proporção de casos e os grupos etários mais acometidos variaram conforme região.

\section{Colaboradores}

M. C. Tauil contribuiu com o desenho do estudo, análise dos dados e redação do artigo. T. R. Amorim e G. F. M. Pereira contribuíram com a revisão crítica do artigo. W. N. Araújo contribuiu com o desenho do estudo e revisão crítica do artigo.

\section{Agradecimentos}

Ao Conselho Nacional de Desenvolvimento Científico e Tecnológico (CNPq), pelo financiamento (processo: 102731/2009-8) durante o Programa de Treinamento em Epidemiologia Aplicada aos Serviços do Sistema Único de Saúde (EPISUS). Aos técnicos da vigilância, informação e pesquisa do Departamento de DST, Aids e Hepatites Virais, em especial ao Silvano Barbosa de Oliveira, pelo suporte na organização e análise dos dados. 


\section{Referências}

1. World Health Organization. Hepatitis B. http:// www.who.int/mediacentre/factsheets/fs204/en/ (acessado em 03/Ago/2010).

2. Yoshida CFT, Gaspar AMC, Lewis-Ximenes LL, Oliveira JM. Hepatites de transmissão parenteral B, Delta e C. In: Coura JR, organizador. Dinâmica das doenças infecciosas e parasitárias. Rio de Janeiro: Editora Guanabara Koogan; 2005. p. 1725-34.

3. Ferreira CT, Silveira TR. Hepatites virais: aspectos da epidemiologia e da prevenção. Rev Bras Epidemiol 2004; 7:473-87.

4. Murray CJL, Lopez AD. Estimating causes of death: new methods and global and regional applications for 1990. In: Murray CJL, Lopez AD, editors. The global burden of disease: a comprehensive assessment of mortality and disability from diseases, injuries, and risk factors in 1990 and project to 2020. Boston:World Health Organization/Harvard School of Public Health/World Bank; 1996. p. 118-200.

5. Wakimoto MD, Marzochi KBF, Hartz ZMA. Avaliação do Sistema de Vigilância Epidemiológica no Município do Rio de Janeiro. In: IV Congresso Brasileiro de Epidemiologia, Livro de Resumos. Rio de Janeiro: ABRASCO; 1998. p. 257.

6. World Health Organization. Hepatitis B. Geneva: World Health Organization; 2002. (WHO/CDS/ CSR/LYO/2002.2).

7. Fonseca JCF. História natural da hepatite crônica B. Rev Soc Bras Med Trop 2007; 40:672-7.

8. Focaccia R, Conceição OJG, Sette Jr. H, Sabino E, Bassit L, Nitrini DR, et al. Estimated prevalence of viral hepatitis in general population of the municipality of Sao Paulo, measured by a serologic survey of a stratified, randomized and residence-based population. Braz J Infect Dis 1998; 2:269-84.

9. Souto F. Distribuição da hepatite viral crônica B no Brasil: atualização do mapa epidemiológico e proposições para seu controle. GED Gastroenterologia e Endoscopia Digestiva 1999; 18:143-9.

10. Viana S. High prevalence of hepatitis B virus and hepatitis D virus in the Western Brazilian Amazon. Am J Trop Med Hyg 2005; 73:808-14.

11. Braga WSM, Brasil LM, Souza RAB, Melo MS, Rosas MDG, Castilho MC, et al. Prevalência da infecção pelos vírus da hepatite viral crônica $\mathrm{B}$ (VHB) e da hepatite Delta (VHD) em Labrea, Rio Purus, Estado do Amazonas. Epidemiol Serv Saúde 2004; 13:35-46.

12. Ministério da Saúde. 30 anos do PNI/CGPNI/ DEVEP/SVS. Brasília: Ministério da Saúde; 2003.

13. Carrazzone CFV, Brito AM, Gomes YM. Importância da avaliação sorológica pré-transfusional em receptores de sangue. Rev Bras Hematol Hemoter 2004; 26:93-8.

14. Núcleo de Pós-Graduação, Universidade de Pernambuco. Estudo de prevalência de base populacional das infecções pelos vírus das hepatites A, B e C nas capitais do Brasil. Dados preliminares do relatório técnico. Recife: Núcleo de Pós-graduação, Universidade de Pernambuco; 2010.

15. World Health Organization. Classificação estatística internacional de doenças e problemas relacionados à saúde: CID 10. São Paulo: Edusp; 1994.
16. Romender JM, McWhinnie JR. Años de vida potencial perdidos entre las edades de 1 y 70 años: un indicador de mortalidad prematura para la planificación de la salud. In: Buck C, organizador. El desafío de la epidemiología. Washington DC: Organización Panamericana de la Salud; 1988.

17. Silva MGC. Anos potenciais de vida perdidos segundo causas, em Fortaleza (Brasil), 1978-80. Rev Saúde Pública 1984; 18:108-21.

18. Bierrenbach AL, Duarte EC, Gomes ABF, Souza MFM. Tendência da mortalidade por tuberculose no Brasil, 1980 a 2004. Rev Saúde Pública 2007; 41 Suppl 1:15-23.

19. Echevarria JM, Leon P. Epidemiology of viruses causing chronic hepatitis among populations from the Amazon Basin and related ecosystems. Cad Saúde Pública 2003; 19:1583-91.

20. Clemens SAC, Da Fonseca JC, Azevedo T, Cavalcanti A, Silveira TR, Castilho MC, et al. Soroprevalência para hepatite A e hepatite B em quatro centros no Brasil. Rev Soc Bras Med Trop 2000; 33:1-10.

21. Braga WSM, Brasil LM, Souza RAB, Castilho MC, Fonseca JC. Ocorrência da infecção pelo vírus da hepatite B (VHB) e delta (VHD) em sete grupos indígenas do Estado do Amazonas. Rev Soc Bras Med Trop 2001; 34:349-55.

22. Oberle MW, Shapiro CN, Lanier AP. Preventing hepatitis B in people in close contact with hepatocellular carcinoma patients. Public Health Rep 1997; 112:63-5.

23. Gonçalves CS, Pereira FEL, Gayotto LCC. Hepatocellular carcinoma in Brazil: report of a national survey (Florianópolis, SC, 1995). Rev Inst Med Trop São Paulo 1997; 39:165-70.

24. Kew MC. Prevention of hepatocellular carcinoma. Ann Hepatol 2010; 9:120-32.

25. Mello-Jorge MHP, Laurenti R, Gotlieb SLD. Análise da qualidade das estatísticas vitais brasileiras: a experiência de implantação do SIM e do SINASC. Ciênc Saúde Coletiva 2007; 12:643-54.

26. Mello-Jorge MHP, Laurenti R, Gotlieb SLD. Avaliação dos sistemas de informação em saúde no Brasil. Cad Saúde Colet (Rio J.) 2010; 18:7-18.

27. Barbosa Júnior A, Szwarcwald CL, Pascom ARP, Souza Júnior PB. Tendências da epidemia de AIDS entre subgrupos sob maior risco no Brasil, 19802004. Cad Saúde Pública 2009; 25:727-37.

28. García-Fulgueiras A, García-Pina R, Morant C, García-Ortuzara V, Génova R, Alvarez E. Hepatitis $\mathrm{C}$ and hepatitis B-related mortality in Spain. Eur J Gastroenterol Hepatol 2009; 21:895-901.

29. Lucena RM, Sousa JL. Anos potenciais de vida perdidos (APVP) por Aids: Pernambuco, 1996 e 2005. DST J Bras Doenças Sex Transm 2009; 21:136-42.

Recebido em 22/Jun/2011

Versão final reapresentada em 26/Out/2011 Aprovado 21/Nov/2011 